\title{
Toward empirical behavior laws: II. Effectiveness of a conditioned reinforcer paired with food and shock
}

\author{
Evalyn F. Segal \\ SAN DIEGO STATE COLLEGE
}

\begin{abstract}
A buzzer was paired with food in control rats and with food and shock in experimentals. (1) Baseline bar pressing in experimentals was depressed. (2) Both groups pressed equally often, on the average, to produce the buzzer. (3) Behavior change was greater in experimentals. The data are consistent with a totalityof-reaction theory of conditioned reinforcement.

Problem

Consider the following hypothesis. The strength of a remforcer is a function of the totality of reaction to it; as the reaction evoked by a stimulus increases, the reinforcing effect is, first, increasingly positive, and then decreasingly positive, and then increasingly negative. Some data in support of the hypothesis were reviewed in another paper (Segal, 1965). Additional support is the following:

(1) The opportunity to engage in one behavior may reinforce other behavior. Fighting cocks were reinforced by the sight of another fighting cock; Siamese fighting fish were more reinforced by the sight of their mirror image, or a moving model of another fish in aggressive display, than by the sight of a stationary model. The reinforcingness of the displays was correlated with their tendency to elicit aggressive reactions in S (Thompson, 1963; cf. Breland \& Breland, 1961).
\end{abstract}

(2) When a link is added to an operant chain, the quantity of behavior that must occur before primary reinforcement arrives increases. If the totality of reaction evoked by stimuli close to food is about optimal, then adding more reaction by adding more links may move the stimulus toward the decreasing limb of the reinforcing-effect function (cf. Segal, 1965). Thus, the stimulus correlated with the earliest link of a long chain may be nonreinforcing, or aversive (Kelleher \& Fry, 1962). Schedule preference studies (e.g., Findley, 1958) also indicate that stimuli associated with large behavior requirements are less positively reinforcing than stimuli associated with smaller behavior requirements. As the requirements progressively rise, the stimuli correlated with the schedule may become aversive (e.g., Appel, 1963; Azrin, 1961).

(3) Pliskoff \& Tolliver (1960) transformed a conditioned positive reinforcer to a conditioned negative reinforcer by depriving food-reinforced rats of water. The $\mathrm{S}^{\mathrm{D}}$ for the food-reinforced operant probably elicited conditioned alimentary respondents which increased in intensity or variety when the $\mathrm{S}^{\mathrm{D}}$ was presented to rats that were water-as well as food-deprived.
As a result, the $\mathrm{S}^{\mathrm{D}}$ became aversive.

(4) A stimulus correlated with a food-reinforcement schedule is more reinforcing when it appears in alternation with a stimulus correlated with extinction, punishment, or a less favorable food-reinforcement schedule (e.g., Reynolds, 1961, 1963; Segal, 1964). The alternation of stimuli may elicit SNS reactions that get conditioned to the preferred stimulus. The combination of $\mathrm{mild}$ SNS reactions and alimentary reactions to food may be more reinforcing than alimentary reactions alone.

(5) A stimulus correlated with extinction or a reduction in reinforcement rate seems to be aversive only if it evokes a large amount of unreinforced operant responding and/or elicits "frustration" reactions (Terrace, 1963a, 1963b, 1964).

Sidman (1960) suggested that a strong test of a hypothesis is the positive verification of a prediction that goes counter to ordinary expectations. An implausible implication of the present theory is that, within limits, increasing the reaction to a stimulus in any way whatever, even by pairing it with shock, will increase its positive reinforcingness. The following experiments attempted to verify this prediction.

\section{Experiment 1}

Method

Two groups of 2 adult, female, albino rats were kept on 23 hours' food deprivation, and run in a conditioning chamber with a floor grid through which $110 \mathrm{~V}$ AC shock, in series with $75 \mathrm{~K}$ resistors, one on each side of the line, could be delivered. Shock was controlled by a manual switch on the chamber operated by E. Food reinforcers were $45 \mathrm{mg}$ Noyes rat pellets. The conditioned reinforcing stimulus was a $24 \mathrm{~V} \mathrm{DC}$ buzzer mounted on the wall of the sound-insulated experimental room. All events except shocks were programmed from another room. E left the room as soon as shock administration was over. Day 1: Buzz-shock pairings. Ss received $203-\mathrm{sec}$. shocks, 2 min. apart, and $204 \mathrm{sec}$. buzzes, 2 min. apart, in a session lasting $40 \mathrm{~min}$. Control rats got shocks and buzzes $60 \mathrm{sec}$. apart. Experimentals got paired stimuli, with shock starting 1 sec. after buzz onset. Operant-level bar presses were noted. (Almost all presses on Day 1 were startle reactions to shock or buzz.) D a y s 2-5: B u z zfood pairings. Ss got 120 pairings daily in 90-min. sessions. Buzz lasted 4 sec.; 1 sec. after its onset, a pellet was dispensed. Buzz onsets were $45 \mathrm{sec}$. apart. Operant level presses were recorded. D a y s 6-9: Conditioned reinforcement test. Bar presses produced 4 sec. of buzz on FR 2. Presses during the buzz did not prolong its duration beyond the programmed $4 \mathrm{sec}$. The first 2 sessions were $2 \mathrm{hrs}$. long, the last $2,1 \mathrm{hr}$.

\section{Results}

The left-most graph in Frame B of Fig. 1 shows the group means of bar presses per 10 min., averaged over all similar sessions. Remaining graphs show data of individual rats and sessions. During the $\mathbf{S}^{\mathbf{r}}$ test, rats of both groups gave about the same number of 


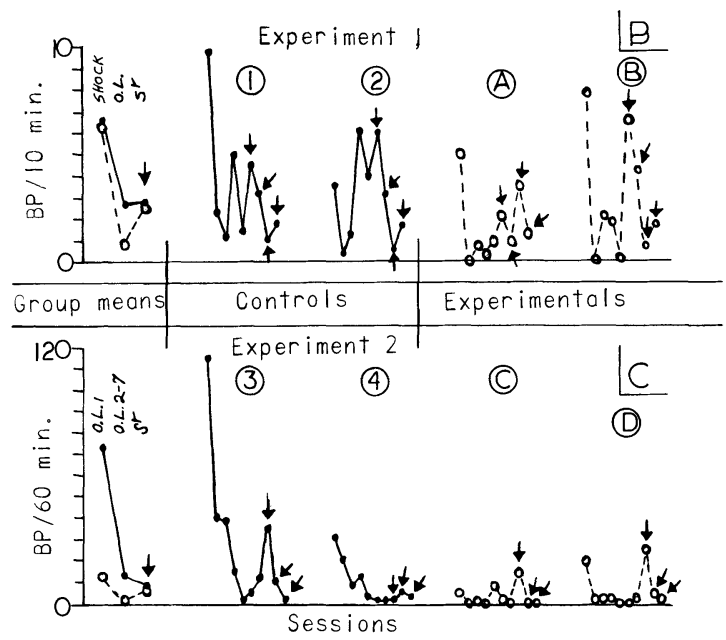

Fig. 1. Frame B: Data from Experiment 1. Frame C: Data from Experiment 2.

responses. However, this represented a much greater increase from operant levels for the experimentals.

\section{Experiment 2}

The lower baseline of the experimentals in Experiment 1 was thought to indicate conditioned suppression elicited by the buzz during collection of operant-level data. In Experiment 2, to reduce conditioned suppression, shocks were spaced one or two per day, fewer total shocks were given, and the bar was removed until all pairings were completed. Then operant levels were noted for several days before conditioned reinforcement testing began.

\section{Method}

Four rats of the same stock, sex, age, and deprivation were used in the same apparatus. Days 1-6: Buzz-shock and buzz-food pairings. On Day 1 , Ss received $23-$ sec. shocks 2 min. apart, and 24 -sec. buzzes $2 \mathrm{~min}$. apart. The stimuli were unpaired in controls, paired in experimentals. Following the second shock, Ss rested 5 min., and then buzz-food pairings began. On Days 2-6, sessions began with 1 , not 2 , shocks and buzzes, and a 2-min., not 5-min., rest followed shock. All sessions were $60 \mathrm{~min}$. long. On Day 1, 25 buzz-food pairings occurred, spaced 2 min. apart. Days 2 \& 4: 100 pairings, 30 sec. apart. Day 3: 55 pairings, 60 sec. apart. Day 5: 75, 45 sec. apart. Day 6: $20,3 \mathrm{~min}$. apart. In all, 7 shocks, paired or unpaired with 7 buzzes, and 395 buzz-food pairings, were given. Days 7-13: Operant level. The bar was replaced, and presses in $60 \mathrm{~min}$. sessions were recorded. Da y s 14-16: C ond ition ed re in for cemen $t$ te st. Presses produced $4 \mathrm{sec}$. of buzz on FR 3 (except that the first press of each session was reinforced). Presses during a buzz did not prolong its duration. Sessions were $60 \mathrm{~min}$.

\section{Results}

The left-most graph in Frame C of Fig. 1 shows the mean group data for all bar-pressing sessions. Operantlevel Day 1 is shown separately from Days 2-7. The remaining graphs show data of individual rats and sessions. As in Experiment 1, both groups gave about the same average number of responses in the reinforcement test. Again, this represented an increase for experimentals, but it represented an average decrease for controls. Only one control, but both experimental Ss showed a significant increase in bar pressing on the first test day.

\section{Diseussion}

The lower baseline of the experimentals in Experiment 2 cannot be conditioned suppression to the buzzer, for the buzzer was not present on operant-level days. Perhaps rats subjected to predictable shock are more anxious, and consequently less active, than rats subjected to unpredictable shock.

By a measure of behavior change (cf Wagman \& Allen, 1964), the buzz was a more effective reinforcer in experimental rats than in controls. By a measure of absolute level of performance, the buzz was equally effective in experimentals and controls. Behavior change seems as important a property of reinforcers as the level of performance they maintain, but even on the more conservative view, the shock-and-food-paired buzz was no less effective a reinforcer than the food-paired buzz. The explanation cannot be that buzzshock pairings were without effect, for they did suppress experimental baselines. The findings are consistent with the totality-ofreaction hypothesis on the supposition that totality of reaction to the food-paired buzz was at a point on the ascending limb of the reinforcing-effect function about level with a point on the descending limb corresponding to the food-and-shock-paired buzz (cf $\underline{x}$ and $\mathrm{y}$ in Fig. 1, Segal, 1965). It might be hard to achieve just that difference in totality of reaction that would place the shock-and-food-paired buzz at a significantly higher positive reinforcingness than the foodpaired buzz. Still, strong confirmation of the hypothesis demands such a demonstration, and efforts tó achieve it are now proceeding.

\section{Referenees}

APPEL, J. B. Aversive aspects of a schedule of positive reinforcement. J. exp. Anal. Behav., 1963, 6, 423-428.

AZRIN, N. H. Time-out from positive reinforcement. Science, 1961, $133,382-383$.

BRELAND, $\mathrm{K}_{\circ}$, \& BRELAND, MARIAN. The misbehavior of organisms. Amer. Psychologist, 1961, 16, 681-684.

FINDLEY, J. D. Preference and switching under concurrent scheduling. J. exp. Anal. Behav., 1958, 1, 123-144.

KELLEHER, R. T., \& FRY, W. Stimulus functions in chained fixedinterval schedules. J. exp. Anal. Behav., 1962, 5, 167-173.

PLISKOFF, S. S., \& TOLLIVER, G. Water-deprivation-produced sign reversal of a conditioned reinforcer based upon dry food. J. exp. Anal. Behav., 1960, 3, 323-329.

REYNOLDS, G. S. Behavioral contrast. J. exp. Anal. Behav., 1961, $4,57-71$.

REYNOLDS, G. S. Potency of conditioned reinforcers based on food and on food and punishment. Science, 1963, 139, 838-839.

SEGAL, EVALYN F. A serial conditioned reinforcement effect in a multiple-chain schedule in a squirrel monkey. Psychon. Sci., $1964,1,175-176$.

SEGAL, EVALYN F. Toward empirical behavior laws: I. Conditioned reinforcement. Psychon. Sci., 1965, 2, 135-136.

SIDMAN, M. Tactics of scientific research. New York: Basic Books, 1960.

TERRACE, H. S. Errorless discrimination learning in the pigeon: Effects of chlorpromazine and imipramine. Science, 1963a, 140, 318-319.

TERRACE, H. S. Discrimination learning with and without "errors." J. exp. Anal. Behav., 1963b, 6, 1-27.

TERRACE, H. S. Wavelength generalization of stimuli correlated with different schedules of reinforcement. Paper read at Psychonomic Society, Niagara Falls, October, 1964.

THOMPSON, T. I. Visual reinforcement in Siamese fighting fish. Science, $1963,141,55-57$.

WAGMAN, W., \& ALLEN, J. D. The development of a conditioned posrtive reinforcer based upon the termination of shock. Psychon. Sci., 1964, 1, 363-364.

Note

1. Supported by NSF G 18132, NSF GB 1605, and NIMH 8505. 